\title{
Optimization of low-frequency low-intensity ultrasound-mediated microvessel disruption on prostate cancer xenografts in nude mice using an orthogonal experimental design
}

\author{
YU YANG, WENKUN BAI, YINI CHEN, YANDUAN LIN and BING HU \\ Department of Ultrasound in Medicine, Shanghai Jiao Tong University Affiliated Sixth People's Hospital, \\ Shanghai Institute of Ultrasound in Medicine, Shanghai 200233, P.R. China
}

Received October 8, 2014; Accepted July 9, 2015

DOI: $10.3892 / \mathrm{ol} .2015 .3716$

\begin{abstract}
The present study aimed to provide a complete exploration of the effect of sound intensity, frequency, duty cycle, microbubble volume and irradiation time on low-frequency low-intensity ultrasound (US)-mediated microvessel disruption, and to identify an optimal combination of the five factors that maximize the blockage effect. An orthogonal experimental design approach was used. Enhanced US imaging and acoustic quantification were performed to assess tumor blood perfusion. In the confirmatory test, in addition to acoustic quantification, the specimens of the tumor were stained with hematoxylin and eosin and observed using light microscopy. The results revealed that sound intensity, frequency, duty cycle, microbubble volume and irradiation time had a significant effect on the average peak intensity (API). The extent of the impact of the variables on the API was in the following order: Sound intensity; frequency; duty cycle; microbubble volume; and irradiation time. The optimum conditions were found to be as follows: Sound intensity, $1.00 \mathrm{~W} / \mathrm{cm}^{2}$; frequency, $20 \mathrm{~Hz}$; duty cycle, $40 \%$; microbubble volume, $0.20 \mathrm{ml}$; and irradiation time, $3 \mathrm{~min}$. In the confirmatory test, the API was $19.97 \pm 2.66$ immediately subsequent to treatment, and histological examination revealed signs of tumor blood vessel injury in the optimum parameter combination group. In conclusion, the Taguchi $\mathrm{L}_{18}(3)^{6}$ orthogonal array design was successfully applied for determining the optimal parameter combination of API following treatment. Under the optimum orthogonal design condition, a minimum API of 19.97 \pm 2.66 subsequent to low-frequency and low-intensity mediated blood perfusion blockage was obtained.
\end{abstract}

Correspondence to: Professor Bing $\mathrm{Hu}$, Department of Ultrasound in Medicine, Shanghai Jiao Tong University Affiliated Sixth People's Hospital, Shanghai Institute of Ultrasound in Medicine, 600 Yi Shan Road, Shanghai 200233, P.R. China

E-mail: hubingedu@hotmail.com

Key words: orthogonal experimental design, low frequency ultrasound, microbubble, prostate carcinoma, tumor vasculature

\section{Introduction}

The morbidity rate of prostate cancer is estimated to be the highest out of the malignancies that occur in American males. This disease is the second leading cause of tumor-associated mortality among American males, accounting for $27 \%$ of all newly diagnosed cancers in men $(n=233,000)$ and $10 \%$ of all male cancer-associated mortalities $(n=29,480)$ in 2014 in the USA (1). Common treatment therapies include radiofrequency ablation, radical prostatectomy, radiation, chemotherapy and active surveillance (2). The majority of patients receive hormone therapy in the early stage of disease, such as androgen-receptor antagonists (3). In the initial stage of disease, the response to androgen deprivation therapy is encouraging, with a significant improvement in clinical symptoms and rapid decline in serum concentrations of prostate specific antigen (PSA) in patients. However, a large proportion of androgen-dependent cancers gradually become resistant to castration and ultimately progress to be more aggressive and androgen-independent, resulting in widespread metastases and an increased incidence of morbidity and mortality $(3,4)$.

Targeting tumor neo-angiogenesis is one of the topics of cancer therapy studies that is increasing the most, as tumors cannot grow beyond $1-2 \mathrm{~mm}^{3}$ in size without a vascular supply (5). The tumor blood supply system acts as an essential factor for tumor growth, progression and metastasis (5). In the absence of angiogenesis, tumor growth is restricted to an extremely small volume, and tumor cells do not spread into the circulation (6). Tumor blood vessels are weaker in structure and less competent compared with the blood vessels of the normal tissues (7). Since the survival and growth of solid tumors depends on tumor angiogenesis and physiological defects in the tumor neovasculature, there is considerable interest in developing techniques that target this neovasculature for cancer therapy. In addition to the numerous pharmacological agents that aim to inhibit or destroy tumor angiogenesis, in previous years there has been an increase in studies investigating a novel anti-vascular approach that uses low-frequency ultrasound and circulating microbubbles (USMB) to disrupt the tumor vasculature (8-10). This therapy has been confirmed to be capable of blocking off tumor blood perfusion without exhibiting an adverse impact on normal tissues. Thus, we 
hypothesize that this technology may be a promising method for the treatment of castration-resistant prostate cancer.

Although there are certain studies that have investigated microbubble parameters, including size or shell properties or ultrasound pulse parameters, including the fundamental transmission frequency, pulse repetition frequency, duty cycle, pulse length, mechanical index and acoustic peak negative acoustic pressure (8,10-13), these studies investigated few parameters simultaneously and did not investigate the different combinations of various levels of parameters. In addition, these studies focused only on the therapeutic effects of USMB, and seldom considered the damage to the skin induced by USMB. Thus, prior to the utilization of USMB in clinical practice, optimization of the therapeutic parameters relative to the blockage of the tumor vasculature is of considerable importance. The orthogonal experimental design is an efficient method for investigating possible influential factors and exploring the optimum combination of therapeutic parameters using a number of experimental sets (14). In an orthogonal design experiment, the levels of all possible factors are selected according to previous literature and preliminary experiments (14). Therefore, the orthogonal experimental design approach has been used for the optimization of a microvessel-disrupting effect followed by a confirmatory experiment.

The present study aimed to use an efficient method to determine the impact of therapeutic parameters on average peak intensity (API) subsequent to treatment and determine the optimum parameter combination. The orthogonal experimental design method may easily reach the present goal. To the best of our knowledge, the present study reports the first successful optimization of multiple parameter combinations of USMB using an orthogonal experimental design.

\section{Materials and methods}

Cell lines and experimental animals. The present study was approved by the Institutional Review Board of the Shanghai Jiao Tong University Affiliated Sixth People's Hospital (Shanghai, China). The human androgen-independent prostate cancer PC3 cell line was obtained from the Cell Bank of the Chinese Academy of Sciences (Beijing, China). The cells were cultured in Dulbecco's modified Eagle's medium (HyClone, Logan, UT, USA) supplemented with $10 \%$ fetal bovine serum (Gibco Life Technologies, Carlsbad, CA, USA) in an incubator with a $5 \% \mathrm{CO}_{2}$ atmosphere at $37^{\circ} \mathrm{C}$. When the cells reached $80 \%$ confluency, they were washed with phosphate-buffered saline, trypsinized and centrifuged at $150 \mathrm{x}$ g for $5 \mathrm{~min}$. Subsequently, the cells were resuspended in phosphate-buffered saline and the final viable cell solution was estimated at a concentration of $1 \times 10^{7}$ cells per $100 \mu 1$.

A total of 27 male Balb-c nude mice (age, 5-6 weeks; weight, 20-25 g) were purchased from Shanghai Super-B\&K Laboratory Animal Corp., Ltd. (Shanghai, China) and raised in the Animal Laboratory of Shanghai Jiao Tong University Affiliated Sixth Peolple's Hospital. The mice were supplied with sufficient chow and water for one week prior to being injected with prostate cancer PC3 cells.

Xenograft tumor model. Following anesthesia by intraperitoneal injection of $50 \mathrm{mg} / \mathrm{kg} 1.5 \%$ pentobarbital sodium and local sterilization, each mouse was then subcutaneously inoculated with $1 \times 10^{7} \mathrm{PC} 3$ cells in the right flank. The mice were raised under specified pathogen-free conditions subsequent to the procedure, and were observed at 2-day intervals. Experiments were initiated 3 weeks later, when the tumors had grown to a maximum diameter of $\sim 10 \mathrm{~mm}$. In total, 18 mice were randomly assigned to the 18 orthogonal experimental trails and 9 mice were randomly assigned to the confirmatory test group.

Therapeutic ultrasound devices. Three low-frequency ultrasound systems manufactured by the Shanghai Institute of Ultrasound in Medicine (Shanghai, China) were used in the present study, with a frequency of 20,80 and $500 \mathrm{kHz}$, respectively. Each device processed the parameters of the three levels, as required by the investigations. With consideration of safety, three levels of low acoustic intensity, consisting of 0.50 , 1.00 and $2.00 \mathrm{~W} / \mathrm{cm}^{2}$, were used to identify the differences between the bioeffects demonstrated by the three acoustic intensities. The ultrasound (US) output was set to a duty cycle of $20 \%$, consisting of $1 \mathrm{sec}$ on and $4 \mathrm{sec}$ off, $40 \%$, consisting of $2 \mathrm{sec}$ on and $3 \mathrm{sec}$ off, and $60 \%$, which consisted of $3 \mathrm{sec}$ on and $2 \mathrm{sec}$ off, respectively. The intermittent working mode allowed microbubbles to refill subsequent to the destruction of each microbubble.

Microbubbles. Albumin-coated microbubbles, termed the perfluoropropane-albumin microsphere injection (Kangrui Pharmaceutical Co., Yueyang, Hunan, China), were used for enhanced US (EUS) imaging and therapeutic application. The microbubble possessed a mean diameter of $3.4 \mu \mathrm{m}$, with $99 \%$ of the particles $<10 \mu \mathrm{m}$ in diameter and a microbubble concentration of $6.5 \times 10^{8}$ microbubbles $/ \mathrm{ml}$. Gentle agitation was performed for $\sim 20 \mathrm{sec}$ to prepare the milky white suspension. For EUS, a bolus injection of $0.10 \mathrm{ml}$ microbubble solution was administered to the mice. For the vessel blocking treatment, a bolus injection of $0.05,0.10$ or $0.20 \mathrm{ml}$ microbubble solution was administered.

Contrast-enhanced US. The Mylab90 US imaging system (Esaote, Genoa, Italy) equipped with a LA522 high frequency linear array probe was used for EUS. Dual-frame imaging combining 2-D and contrast modality was conducted using a low mechanical index (MI; MI, 0.05). Depth, frequency and other US conditions maintained consistency during all EUS studies. The real-time EUS dynamic images were recorded for $3 \mathrm{~min}$ at a time following an intravenous bolus injection of the microbubbles. The dynamic images were stored in the built-in hard disk of the machine and then exported for contrast analysis.

Image analysis. The EUS images were analyzed using the QontraXt software (Esaote) to identify the average peak contrast enhancement of tumor perfusion. The QontraXt software quantification interface exhibited the 2-D and EUS images. The boundary of the tumor was utilized as the region of interest (ROI), and no marked breathing movement was noted during EUS. The first 3 min was defined and then a time-intensity curve was automatically generated. The parameters that indicated the degree of tumor blood perfusion, and 
Table I. Levels and factors of orthogonal design.

Factor

\begin{tabular}{lccccc}
\cline { 2 - 5 } Level & $\begin{array}{c}\text { Sound intensity, } \\
\text { W/cm }\end{array}$ & Frequency, Hz & Duty cycle, \% & $\begin{array}{c}\text { Microbubble } \\
\text { volume, } \mathrm{ml}\end{array}$ & $\begin{array}{c}\text { Irradiation } \\
\text { time, min }\end{array}$ \\
\hline 1 & 0.50 & 20 & 20 & 0.05 & 1 \\
2 & 1.00 & 80 & 40 & 0.10 & 3 \\
3 & 2.00 & 500 & 60 & 0.20 & 5 \\
\hline
\end{tabular}

therefore API, were calculated automatically. The API represents the average peak intensity or peak acoustic intensity, which is the average echogenicity within a ROI at the peak contrast enhancement.

Treatment protocols. A six-factor three-level orthogonal design that included a blank column $\left[\mathrm{L}_{18}(3)^{6}\right]$ was employed for investigating the effects of the therapeutic parameters on the API subsequent to treatment. The therapeutic parameters were the sound intensity, frequency, duty cycle, microbubble volume and irradiation time. The therapeutic parameters are reported in Table I.

All 18 tumors were first imaged by 2-D US and EUS $1 \mathrm{~h}$ prior to treatment. Following an intravenous injection of microbubbles via the tail veins, the therapeutic transducer coupled with gel was applied to the tumor percutaneously. The treatment lasted for 1,3 or $5 \mathrm{~min}$, with duty cycles of 20 , 40 and $60 \%$. This was followed by the injection of $0.05,0.10$ or $0.20 \mathrm{ml}$ microbubble solution. The diameter of the therapeutic US transducer was $20 \mathrm{~mm}(20 \mathrm{kHz}), 13 \mathrm{~mm}(80 \mathrm{kHz})$ and $13 \mathrm{~mm}(500 \mathrm{kHz})$ respectively, which covered the entire tumor. Following the procedure, EUS was immediately performed, and then all mice were euthanized by the administration of an overdose injection of sodium pentobarbital $(150 \mathrm{mg} / \mathrm{kg})$.

In total, 9 nude mice were assigned in the confirmatory test, with 3 mice in each group, which consisted of the optimum combination in intuitive result, optimum combination in variance analysis and control groups. In addition to the aforementioned procures, mice from each group were euthanized and the tumors were removed and prepared for additional histological examination.

Histological examination. At the end of treatment, all mice were sacrificed and the tumors were surgically excised. The tumor specimens were harvested and fixed in $10 \%$ formaldehyde for $24 \mathrm{~h}$. The tissues were then embedded in paraffin and $5-\mu \mathrm{m}$ thick sections of the tissues were stained with hematoxylin and eosin (HE). A pathologist blinded to the study morphologically evaluated the tissue sections using a light microscope (CX41; Olympus, Tokyo, Japan).

Statistical analysis. All data are expressed as the mean \pm standard deviation. SPSS 19.0 software (IBM, Armonk, NY, USA) was used to analyze the data. One-way analysis of variance (ANOVA) was used for comparisons of differences between groups in confirmatory test. The least significant difference test was used for multiple comparisons.
$\mathrm{P}<0.05$ was considered to indicate a statistically significant difference.

\section{Results}

Xenograft tumor model. The tumors were established in all animals following inoculation with PC-3 cells. The implanted tumors were observed to be spherical, elliptical or nodular by US. All animals survived the procedures until the mice were sacrificed at a designated time-point subsequent to the procedure.

Intuitive results. In total, 18 experiments were designed, according to the $\mathrm{L}_{18}(3)^{6}$ orthogonal array, and the corresponding API values were obtained and listed in Table II. The values chosen for each level were based on the literature $(10,15,16)$ and preliminary tests. In the 18 tests, various EUS perfusion changes were observed from the API quantification (Table II). Certain tumors revealed rich and torch-like enhancement in the contrast arterial phase, which was usually 8-15 sec subsequent to microbubble injection, and others exhibited dotted or nodular enhancement, particularly in the tumor periphery. Certain tumors also revealed completely deficient perfusion from the direct view.

The experimental data obtained from the orthogonal design are reported in Table III, with $\mathrm{k}$ being the average total API for certain factors at one level, the range value (R) being $\mathrm{k}_{\max }-\mathrm{k}_{\min }$, and $\mathrm{k}_{\max }$ and $\mathrm{k}_{\min }$ being the maximum and minimum values of $\mathrm{k}_{1}, \mathrm{k}_{2}$ and $\mathrm{k}_{3}$, respectively. The factor with the largest range value demonstrated the greatest effect on the API. Table III reports that the rank order of the five influential factors is sound intensity, frequency, duty cycle, microbubble volume and irradiation time. The minimum API was obtained using the A3B1C3D3E3 parameters, which consisted of a sound intensity of $2.00 \mathrm{~W} / \mathrm{cm}^{2}$, frequency of $20 \mathrm{~Hz}$, duty cycle of $60 \%$, microbubble volume of $0.20 \mathrm{ml}$ and irradiation time of $5 \mathrm{~min}$.

Results of variance analysis. The results of ANOVA are reported in Table IV. All factors demonstrated a significant effect $(\mathrm{P}<0.05)$ on API within the studied range. Table IV reports that the F-value of the five influential factors was in the following order: Sound intensity; frequency; duty cycle; microbubble volume; and irradiation time. The response curves of the sound intensity, frequency, duty cycle, microbubble volume and irradiation on API are exhibited in Figs. 1-5. As the sound intensity increased, the API subsequent to treatment declined, 
Table II. The experimental design based on L18 $\left(3^{6}\right)$ orthogonal array and experimental results.

\begin{tabular}{|c|c|c|c|c|c|c|c|}
\hline \multirow[b]{2}{*}{ Number } & \multicolumn{6}{|c|}{ Factor } & \multirow[b]{2}{*}{ API } \\
\hline & $\begin{array}{l}\text { Sound intensity, } \\
\mathrm{W} / \mathrm{cm}^{2}\end{array}$ & $\begin{array}{c}\text { Frequency, } \\
\mathrm{Hz}\end{array}$ & $\begin{array}{c}\text { Duty } \\
\text { cycle, \% }\end{array}$ & $\begin{array}{c}\text { Microbubble } \\
\text { volume, ml }\end{array}$ & $\begin{array}{l}\text { Irradiation } \\
\text { time, min }\end{array}$ & $\begin{array}{l}\text { Blank } \\
\text { column }\end{array}$ & \\
\hline 1 & 1 & 1 & 1 & 1 & 1 & 1 & 63.1 \\
\hline 2 & 1 & 1 & 3 & 3 & 2 & 2 & 40.5 \\
\hline 3 & 1 & 2 & 1 & 1 & 3 & 3 & 57.6 \\
\hline 4 & 1 & 2 & 2 & 2 & 2 & 2 & 52.3 \\
\hline 5 & 1 & 3 & 2 & 2 & 1 & 1 & 68.7 \\
\hline 6 & 1 & 3 & 3 & 3 & 3 & 3 & 49.8 \\
\hline 7 & 2 & 1 & 1 & 2 & 2 & 3 & 38.5 \\
\hline 8 & 2 & 1 & 2 & 3 & 1 & 3 & 27.1 \\
\hline 9 & 2 & 2 & 2 & 3 & 3 & 1 & 33.1 \\
\hline 10 & 2 & 2 & 3 & 1 & 2 & 1 & 34.1 \\
\hline 11 & 2 & 3 & 1 & 2 & 3 & 2 & 54.6 \\
\hline 12 & 2 & 3 & 3 & 1 & 1 & 2 & 53.7 \\
\hline 13 & 3 & 1 & 2 & 1 & 3 & 2 & 31.6 \\
\hline 14 & 3 & 1 & 3 & 2 & 3 & 1 & 18.0 \\
\hline 15 & 3 & 2 & 1 & 3 & 1 & 2 & 43.6 \\
\hline 16 & 3 & 2 & 3 & 2 & 1 & 3 & 35.1 \\
\hline 17 & 3 & 3 & 1 & 3 & 2 & 1 & 41.0 \\
\hline 18 & 3 & 3 & 2 & 1 & 2 & 3 & 46.2 \\
\hline
\end{tabular}

API, average peak intensity.

Table III. Results of intuitive analysis.

\begin{tabular}{lcccrrr}
\hline Value & $\begin{array}{c}\text { Sound intensity, } \\
\mathrm{W} / \mathrm{cm}^{2}\end{array}$ & Frequency, $\mathrm{Hz}$ & Duty cycle, $\%$ & $\begin{array}{c}\text { Microbubble } \\
\text { volume, ml }\end{array}$ & $\begin{array}{c}\text { Irradiation } \\
\text { time, min }\end{array}$ & $\begin{array}{c}\text { Blank column } \\
\mathrm{K} 1\end{array}$ \\
$\mathrm{~K} 2$ & 332.0000 & 218.8000 & 298.4000 & 286.3000 & 291.3000 & 258.0000 \\
$\mathrm{~K} 3$ & 241.1000 & 255.8000 & 259.0000 & 267.2000 & 252.6000 & 276.3000 \\
$\mathrm{k}_{1}$ & 215.5000 & 314.0000 & 231.2000 & 235.1000 & 244.7000 & 254.3000 \\
$\mathrm{k}_{2}$ & 55.3333 & 36.4667 & 49.7333 & 47.7167 & 48.5500 & 43.0000 \\
$\mathrm{k}_{3}$ & 40.1833 & 42.6333 & 43.1667 & 44.5333 & 42.1000 & 46.0500 \\
$\mathrm{R}$ & 35.9167 & 52.3333 & 38.5333 & 39.1833 & 40.7833 & 42.3833 \\
\hline
\end{tabular}

$\mathrm{K}$, Sum of API for the factors at each level; $\mathrm{k}$, The mean values of API for the factors at each level; $\mathrm{R}, \mathrm{k}_{\max }-\mathrm{k}_{\min }$.

but multiple comparisons revealed that the difference between $1.00 \mathrm{~W} / \mathrm{cm}^{2}$ and $2.00 \mathrm{~W} / \mathrm{cm}^{2}$ was not statistically significant $(\mathrm{P}=0.102)$. As the frequency increased, the API subsequent to treatment also increased, and the difference between each frequency was significant $(\mathrm{P}<0.05)$. The API declined as the duty cycle increased, but the difference between the 40 and $60 \%$ duty cycles was not significant $(\mathrm{P}=0.081)$. The API decreased as the microbubble volume increased, but only the difference between microbubble volumes of 0.05 and $0.20 \mathrm{ml}$ was statistically significant $(\mathrm{P}=0.007)$. The API decreased as the irradiation time increased, but the difference between 3 and 5 min was not significant $(\mathrm{P}=0.580)$. Therefore, the minimum
API was obtained using the A2B1C2D3E2 parameters, which consisted of a sound intensity of $1.00 \mathrm{~W} / \mathrm{cm}^{2}$, frequency of $20 \mathrm{~Hz}$, duty cycle of $40 \%$, microbubble volume of $0.20 \mathrm{ml}$ and irradiation duration of $3 \mathrm{~min}$.

API analysis of verification experiment. As the combinations of factors identified as optimal following calculation, which consisted of the A3B1C3D3E3 parameters as the intuitive result and the $\mathrm{A} 2 \mathrm{~B} 1 \mathrm{C} 2 \mathrm{D} 3 \mathrm{E} 2$ parameters from variance analysis, had not been tested previously and the ANOVA result was not in agreement with the intuitive result, it was necessary to perform a confirmatory experiment to probe the reliability of 
Table IV. Results of variance analysis.

\begin{tabular}{lccccc}
\hline Source & Sum of squares & df & F-value & P-value & Significance \\
\hline Sound intensity, W/cm & 1249.468 & 2 & 40.353 & 0.000 & $<0.001$ \\
Frequency, Hz & 767.738 & 2 & 24.795 & 0.001 & $<0.05$ \\
Duty cycle, \% & 380.058 & 2 & 12.274 & 0.005 & $<.05$ \\
Microbubble volume, ml & 223.148 & 2 & 7.207 & 0.024 & $<0.05$ \\
Irradiation time, min & 207.314 & 2 & 6.695 & & \\
Blank column (Error) & 108.372 & 7 & & & \\
\hline
\end{tabular}

df, Degrees of freedom.

Table V. Verification experimental results.

\begin{tabular}{lcc}
\hline Group & API & Injury \\
\hline A2B1C2D3E2 & $19.97 \pm 2.66^{\mathrm{a}}$ & No \\
A3B1C3D3E3 & $18.47 \pm 0.81^{\mathrm{a}}$ & Yes \\
Control & $72.37 \pm 3.84$ & \\
\hline
\end{tabular}

A2B1C2D3E2, sound intensity of $1.00 \mathrm{~W} / \mathrm{cm}^{2}$, frequency of $20 \mathrm{kHz}$, duty cycle of $40 \%$, microbubble volume of $0.20 \mathrm{ml}$, irradiation time of $3 \mathrm{~min}$; A3B1C3D3E3, sound intensity of $2.00 \mathrm{~W} / \mathrm{cm}^{2}$, frequency of $20 \mathrm{kHz}$, duty cycle of $60 \%$, microbubble volume of $0.20 \mathrm{ml}$, irradiation time of $5 \mathrm{~min}$; API, average peak intensity. ${ }^{\text {a }} \mathrm{P}<0.05 \mathrm{vs}$. control.

the results obtained. The experimental results are reported in Table V. In the controls, the contrast enhancement in all tumors began at $8-15$ sec subsequent to microbubble injection and rapidly spread from the periphery to the tumor center, and all tumors demonstrated abnormal, rich and torch-like enhancement (Fig. 6), although irregular and patchy non-enhanced regions occasionally occurred at the tumor center.

In the two treatment groups, the EUS results revealed that the circulation of prostatic tumors may be completely blocked in the treated tumors. There was a significantly decreased API in each of the two experimental groups compared with the controls. Immediately subsequent to the treatment, the tumor contrast perfusion completely disappeared, and a significant perfusion defect was formed covering the targeted tumor in each of the two treatment groups (Table V), with an API of $19.97 \pm 2.66$ in the A2B1C2D3E2 group, and the A3B1C3D3E3 group with API of $18.47 \pm 0.81$. There was no statistically significant difference between the A2B1C2D3E2 group and A3B1C3D3E3 group, but skin injury was observed in the A3B1C3D3E3 group, comprising skin burns, skin breaks and bleeding, while there was no evident skin change in the A2B1C2D3E2 group.

Histological findings of the verification experiment. On the HE-stained slides, at low magnification (x100), PC3 prostate tumor cells in the control group were exhibited as an invasive cancer nest. The tumor demonstrates a typical histological pattern characterized by loosely spaced and cord-like neoplastic cells forming a mass with reduced connective tissues and a sharply demarcated encapsulated border. At a high magnification (x400), the tumor cell nuclei were large and deeply stained, with a large karyoplasmic ratio and increased karyokinesis. Tumor necrosis was rare and not evident. The clear blood vessels were almost unobservable, although occasionally microvessels with a few red blood cells in the lumen were observed.

In the two treatment groups, disruption of the tumor vasculature and severe vessel dilation were identified in the HE-stained sections of 6 tissue specimens subsequent to treatment (Fig. 7). The aforementioned histological findings in the A2B1C2D3E2 and A3B1C3D3E3 groups appeared similar, but these changes tended to be distributed slightly more diffusely and more severe in the A3B1C3D3E3 group compared with the A2B1C2D3E2 group. The vascular wall construction of the tumor tissues was disrupted, resulting in a near disappearance of the blood wall structure and thrombosis and hematoma formation distributed outside the vessel (Fig. 7C). The tumor microvasculature revealed severe microvessel dilation (Fig. 7D) accompanied by intravascular thrombosis (Fig. 7E). Edema was frequently observed in the vicinity of the ruptured vessels, and the tumor cells were widely separated by edema (Fig. 7E). Multiple pseudocysts in irregular round or oval appearances of various sizes were frequently observed. The cysts were delineated without endothelial lining around the border (Fig. 7E and F). Despite the vascular changes, the majority of the individual neoplastic cells appeared normal and alive. However, due to the large amount of fluid exudation to the outside of the blood vessel and a large number of interstitial cavities formed, certain tumor cells near disrupted vessels were pressed, and their volume was reduced (Fig. 7E).

\section{Discussion}

Ultrasound contrast agent microbubbles have gained considerable attention due to their clinical application in US contrast imaging and potential therapeutic effects (17). The microbubbles contain gas encased in a shell, and they are capable of passing through the capillary lumen. Various studies have revealed that microbubbles may induce significant effects on confining vasculature (18-20). The effects include increasing the vascular permeability for chemotherapy drugs and target gene delivery, opening the blood-brain barrier locally and transiently, and also vessel rupture and occlusion $(9,10,13,21-25)$. Tumor blood perfusion may be blocked to various degrees 


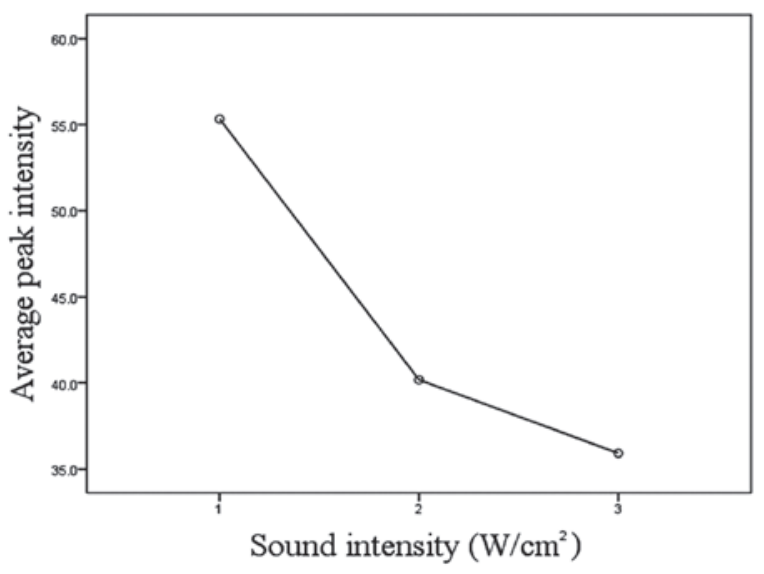

Figure 1. Effect of sound intensity on the average peak intensity. 1, $0.50 \mathrm{~W} / \mathrm{cm}^{2} ; 2,1.00 \mathrm{~W} / \mathrm{cm}^{2} ; 3,2.00 \mathrm{~W} / \mathrm{cm}^{2}$.

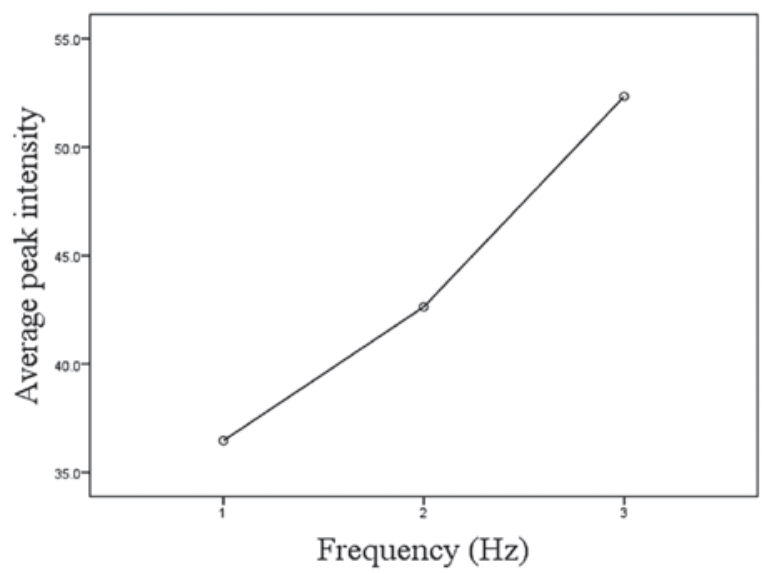

Figure 2. Effect of frequency on the average peak intensity. 1, $20 \mathrm{kHz} ; 2$, $80 \mathrm{kHz} ; 3,500 \mathrm{kHz}$.

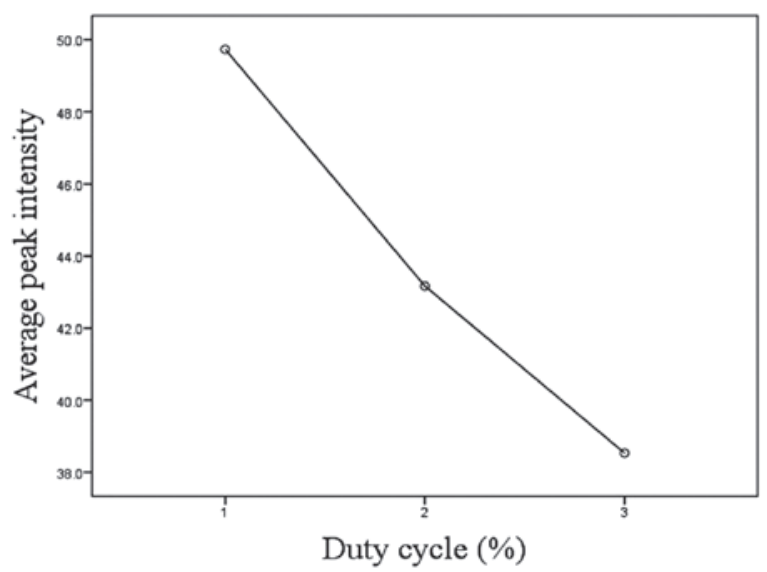

Figure 3. Effect of duty cycle on the average peak intensity. $1,20 \%$ (1 sec on and $4 \mathrm{sec}$ off); $2,40 \%$ ( $2 \mathrm{sec}$ on and $3 \mathrm{sec}$ off); $3,60 \%$ (3 sec on and $2 \mathrm{sec}$ off).

using high-intensity focused US (HIFU) and low-frequency US $(10,26,27)$. With the presence of microbubbles, this vascular disruption effect may be performed at a low acoustic intensity, and it overcomes the thermal adverse effects of HIFU ablation (15).

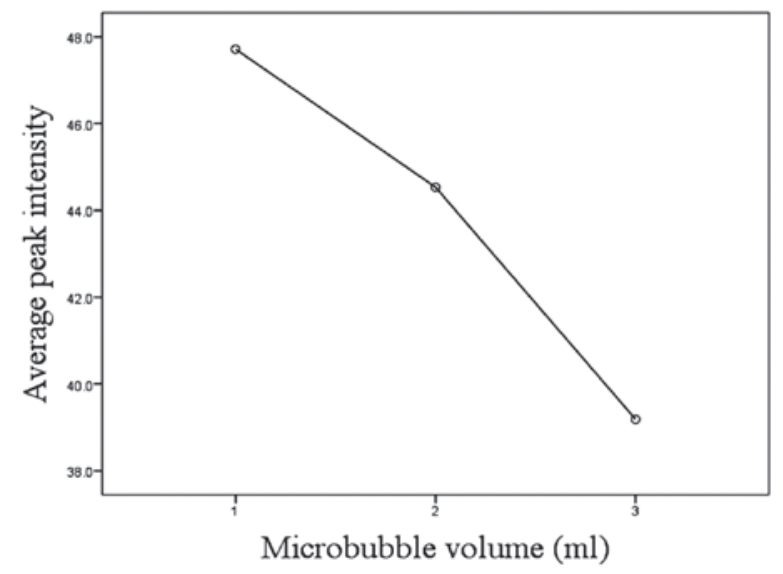

Figure 4. Effect of microbubble volume on the average peak intensity. 1 , $0.05 \mathrm{ml} ; 2,0.10 \mathrm{ml} ; 3,0.20 \mathrm{ml}$.

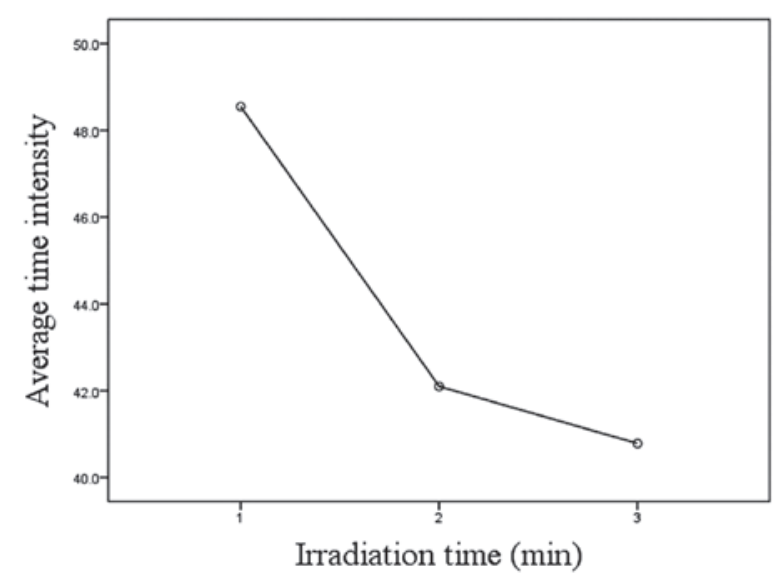

Figure 5. Effect of irradiation time on the average peak intensity. 1, 1 min; $2,3 \mathrm{~min} ; 3,5 \mathrm{~min}$.

The anti-vascular effect of USMB is promising and reproducible $(5,10,11,28)$. It has been demonstrated that thermal, mechanical, such as cavitation and other non-linear mechanisms, and sonochemical effects are likely to contribute to the anti-vascular activities of microbubbles (7). These acoustic mechanisms may act either to directly or indirectly damage vascular structure by inducing tissue response to the thermal, mechanical or sonochemical effects (7). Inertial cavitation and its corresponding effects are considered to be the dominating mechanism for microvessel damage $(10,29)$. Acoustic cavitation is defined as the formation, growth, oscillation and collapse of bubbles under the effect of an ultrasonic wave in a liquid medium (30). Acoustic cavitation is divided into stable and transient cavitation. Bubbles pulsating over numerous acoustic propagation cycles without collapse is classified as stable cavitation, whereas transient cavitation is classified as intense and fast bubble expansion over several acoustic wave cycles, leading to the eventual collapse into smaller bubbles $(31,32)$. In the US field, when bubbles pass through blood vessels, the bubbles grow and contract in response to the acoustic wave, in accordance with the changing acoustic pressure over time, and these volumetric oscillations are important in their effect on the therapeutic process (33). In stable cavitation, the oscillation of 
A

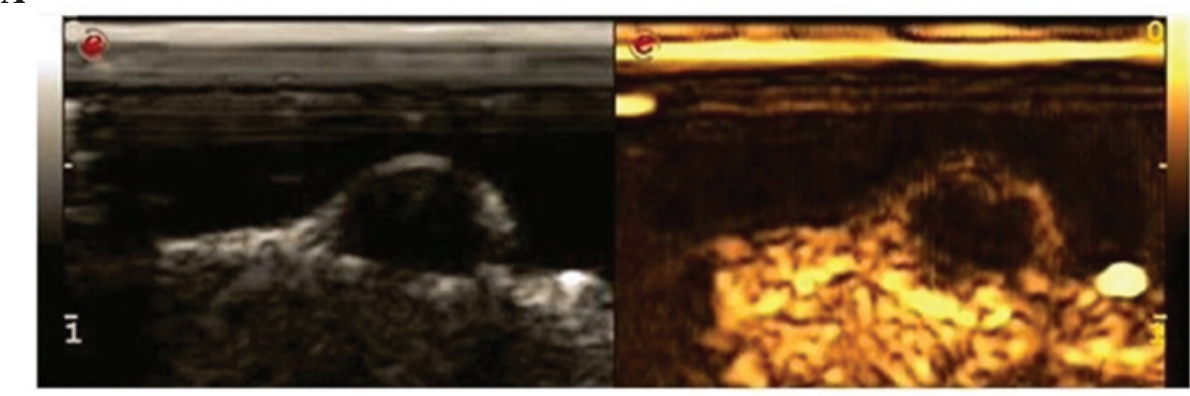

B

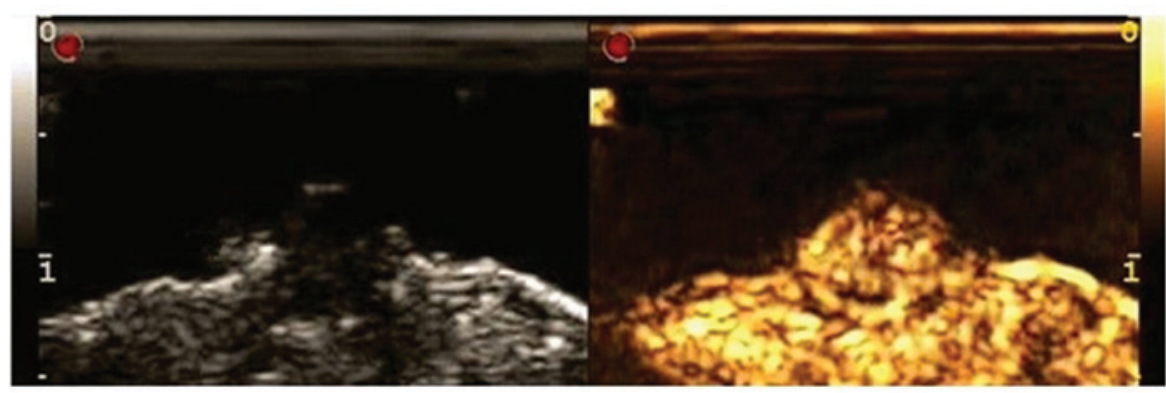

Figure 6. Results of 2-D and EUS imaging of the A2B1C2D3E2 and control groups. (A) The 2-D and EUS images of a subcutaneous tumor in the A2B1C2D3E2 group, demonstrating that a significant tumor perfusion defect occurred. (B) The 2-D and EUS images of a tumor in the control group, demonstrating an abundant perfusion enhancement of tumor vasculature. 2-D, two-dimensional; EUS, enhanced ultrasound; A2B1C2D3E2, sound intensity of $1.00 \mathrm{~W} / \mathrm{cm}^{2}$, frequency of $20 \mathrm{kHz}$, duty cycle of $40 \%$, microbubble volume of $0.20 \mathrm{ml}$ and irradiation time of $3 \mathrm{~min}$.
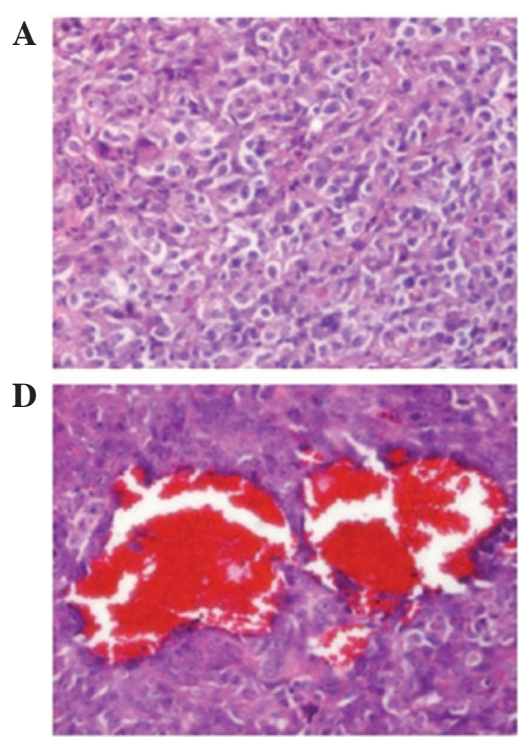
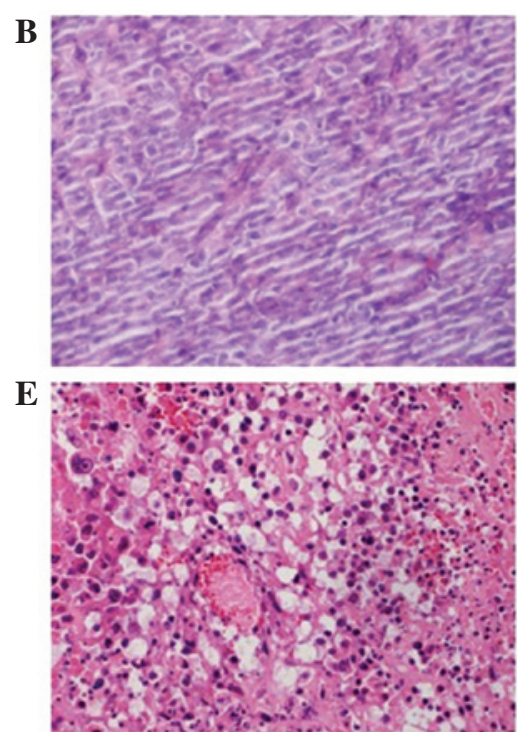
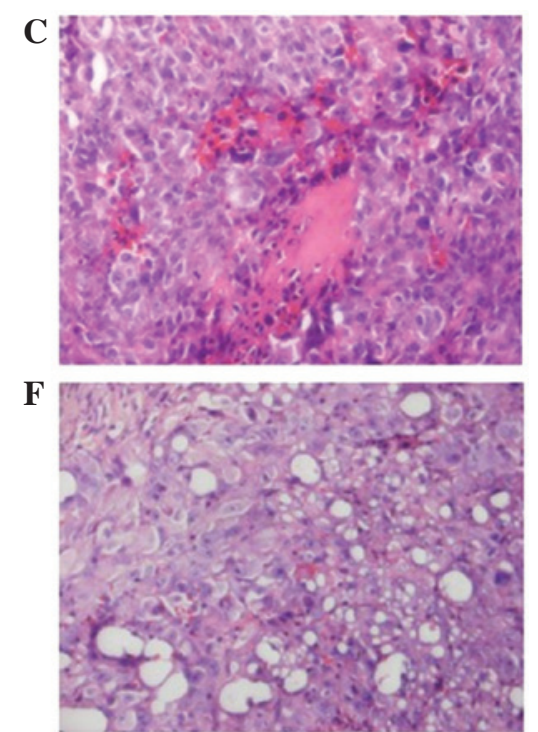

Figure 7. Histological findings of the control and A2B1C2D3E2 groups. Pathology of the prostate cancer PC3 tumor tissues of nude mice, determined using light microscopy, in the (A and B) control group and (C-F) A2B1C2D3E2 groups (magnification, x400). (A and B) The untreated neoplasms demonstrated distinct, unencapsulated margins and were characterized by loosely spaced and cord-like PC3 cells. The A2B1C2D3E2 group demonstrated that the tumor microvasculature was disrupted into (C) diffuse hematomas, (D) severe microvessel dilation accompanied by (E) thrombosis, (E) intercellular edema and (E and F) the formation of multiple cysts of various sizes. A2B1C2D3E2, sound intensity of $1.00 \mathrm{~W} / \mathrm{cm}^{2}$, frequency of $20 \mathrm{kHz}$, duty cycle of $40 \%$, microbubble volume of $0.20 \mathrm{ml}$ and irradiation time of $3 \mathrm{~min}$.

the bubbles may trigger the surrounding fluid to flow near the pulsating bubbles, termed microstreaming (34). Microstreaming imposes considerable shear stress on nearby vascular epithelial cells, and at high enough stresses, these endothelial cells may eventually be detached from the vessel wall (35). In addition, in stable cavitation, the radiation force may push bubbles in the direction of wave propagation, which may exert an effect on the endothelium (36). Furthermore, in the transient cavitation stage, the oscillation of the bubbles becomes so energetic that the bubbles eventually collapse, inducing a high local temperature and pressure, and producing free radicals in the surrounding blood (37). Overall, these effects result in a shock wave. If microbubbles collapse near a blood vessel wall, the shock wave may create liquid jets that affect the vessel, creating holes in the vessel wall (38). Therefore, the expansion, oscillation and collapse of the bubbles and the resultant microstreaming, shock wave and fluid jets altogether result in microvessel distention, invagination and ultimately vessel rupture $(12,18,20,29,38)$. 
The factors associated with cavitation include the US intensity, frequency, duty cycle and microbubble numbers or concentration in the target vessel. In the present study, the microbubble injection volume was investigated. According to the range value of intuitive result and F-value of the ANOVA result, the extent of the impact of the variables on the API was determined to be in the following order: Sound intensity; frequency; duty cycle; microbubble volume; and irradiation time. Ultrasound intensity exerted a notable impact on vessel damage, particularly when exogenous US imaging microbubbles are present (8). The present study revealed that, as the sound intensity increased, the API subsequent to treatment declined, which is consistent with the study by Liu et al (10). This may be due to microbubbles being able to undergo more severe vibration at a higher intensity, thus transient cavitation is more intense. However, the difference between the $1.00 \mathrm{~W} / \mathrm{cm}^{2}$ and $2.00 \mathrm{~W} / \mathrm{cm}^{2}$ sound intensities was not significant. This result demonstrates that $1 \mathrm{~W} / \mathrm{cm}^{2}$ is sufficient in inducing the vascular disrupting effect.

Low-frequency US is usually defined as US with frequencies in the range of $20-100 \mathrm{kHz}(30,31)$. In order to investigate the effect of frequency on USMB more deeply, a frequency of $500 \mathrm{kHz}$ was incorporated into the present study. As the frequency increased, the API following treatment increased and multiple comparisons revealed that the difference between each comparison was significant. This result is consistent with the general finding that the inertial cavitation activity is produced with decreasing acoustic frequency $(39,40)$. At lower frequencies, the growth phase of the bubble is longer and the bubble radius therefore increases. Consequently bubble elapse will demonstrate a more significant effect (34). In the present study, an intermittent working mode was used to allow microbubbles to refill subsequent to the destruction of all microbubbles. The API decreased as the duty cycle increased, although multiple comparisons revealed that the difference between the 40 and $60 \%$ duty cycles was not significant. This result confirmed the necessity of microbubble reperfusion. When the duty cycle was $20 \%$, the block-off effect is impaired, as spatial peak temporal average intensity is decreased. The blocking effect was no longer reinforced when the duty cycle was increased between 40 and $60 \%$, which revealed that a duty cycle of $40 \%$ is sufficient for reperfusion, and no additional increase is required. As for exogenous microbubble volume, the API decreased as the microbubble volume increased, although only the difference between $0.05 \mathrm{ml}$ and $0.20 \mathrm{ml}$ was statistically significant. Therefore, in clinical practice, on the premise of safety, more microbubbles should be injected to achieve a greater therapeutic effect.

Whether inertial cavitation occurred in the present study has not yet been elucidated. If inertial cavitation were to occur, it may also contribute to anti-vascular activity. A previous in vivo study (41), led to the half-life of inertial cavitation being estimated as only $20 \mathrm{sec}$ in duration. The insonation time used in the present study was more than a few seconds, and as the irradiation time increased, the API decreased, although the difference between treatment for $3 \mathrm{~min}$ and $5 \mathrm{~min}$ was not significant. This indicates that cavitation may not be an exclusive factor in USMB, and the participation of heating in the therapeutic process cannot be excluded. Heating is produced by the absorption of US energy through viscous damping of oscillating microbubbles (7). The network of tumor blood vessels is disorganized and tortuous and is likely to lead to the localized entrapment of microbubbles (7). As a result, the microbubbles are likely to reside in the US beam longer in the tumor tissue compared with the normal tissue. Thus, microbubble-induced heating may result in increased temperatures in the cancerous tissue compared to those in the healthy tissue. In addition, the blood vessel blockage caused in the targeted regions may result in increased heating. This temperature differential in tissues makes it possible to selectively disrupt tumor blood vessels, without causing a significant adverse effect on normal blood vessels (7). Therefore, it may be hypothesized that the thermal effect is an important component of targeting the tumor neovasculature.

In the confirmatory test group, pathological changes in the tumors confirmed the vascular effects detected using EUS. The dilatation of microvessels, interstitial hemorrhage and thrombosis may be due to microvascular rupture. Blood extravasation and compression resulted in the compression of tumor cells, with interstitial edema in the vicinity of the blood vessels. These changes may form due to the combined pressure from microcirculation, which may slow down and even block blood perfusion to the tumor. The API obtained under optimal conditions was low $(19.97 \pm 2.66)$ compared with the controls, indicating poor blood perfusion, which confirmed the efficacy of the orthogonal experimental design in identifying the impact of factors on API and determining the optimum parameter combination. The API standard deviations were small in contrast to the average API values. This may indicate the reproducibility of the vascular effects induced by the vessel-blocking treatment.

In conclusion, the $\mathrm{L}_{18}(3)^{6}$ orthogonal array design was successfully applied to determine the optimum parameter combination. The extent of the impact of variables on the API was determined to be in the following order: Sound intensity; frequency; duty cycle; microbubble volume; and irradiation time. It was also concluded that the optimum parameter combination is A2B1C2D3E2, which consisted of a sound intensity of $1.00 \mathrm{~W} / \mathrm{cm}^{2}$, frequency of $20 \mathrm{kHz}$, duty cycle of $40 \%$, microbubble volume of $0.20 \mathrm{ml}$ and irradiation time of $3 \mathrm{~min}$. This results in good repeatability, without skin injury. In conclusion, a minimum API of $19.97 \pm 2.66$ subsequent to USMB-mediated blood perfusion blockage was obtained using the optimum orthogonal design. The optimum parameter combination achieved in the present study may provide evidence to support the use of USMB in the treatment of prostate cancer in the future.

\section{Acknowledgements}

The authors would like to thank Kangrui Pharmaceutical Corporation for providing the perfluoropropane-albumin microsphere injection. This study was supported by the National Natural Science Foundation of China (grant nos. 81271597 and 81401421).

\section{References}

1. Siegel R, Ma J, Zou Z and Jemal A: Cancer statistics, 2014. CA Cancer J Clin 64: 9-29, 2014. 
2. Fung-Kee-Fung SD, Porten SP, Meng MV and Kuettel M: The role of active surveillance in the management of prostate cancer. J Natl Compr Canc Netw 11: 183-187, 2013.

3. Goldberg AA, Titorenko VI, Beach A and Sanderson JT: Bile acids induce apoptosis selectively in androgen-dependent and -independent prostate cancer cells. PeerJ 1: e122, 2013.

4. Pienta KJ and Smith DC: Advances in prostate cancer chemotherapy: A new era begins. CA Cancer J Clin 55: 300-318, 2005.

5. El Kaffas A, Nofiele J, Giles A, Cho S, Liu SK and Czarnota GJ Dll4-notch signalling blockade synergizes combined ultrasound-stimulated microbubble and radiation therapy in human colon cancer xenografts. PloS one 9: e93888, 2014.

6. Folkman J: Angiogenesis and apoptosis. Semin Cancer Biol 13: 159-167, 2003.

7. Levenback BJ, Sehgal CM and Wood AK: Modeling of thermal effects in antivascular ultrasound therapy. J Acoust Soc Am 131: 540-549, 2012.

8. Hwang JH, Brayman AA, Reidy MA, Matula TJ, Kimmey MB and Crum LA: Vascular effects induced by combined $1-\mathrm{MHz}$ ultrasound and microbubble contrast agent treatments in vivo. Ultrasound Med Biol 31: 553-564, 2005.

9. Wood AK, Bunte RM, Price HE, Deitz MS, Tsai JH, Lee WM and Sehgal CM: The disruption of murine tumor neovasculature by low-intensity ultrasound-comparison between 1- and 3-MHz sonication frequencies. Acad Radiol 15: 1133-1141, 2008.

10. Liu Z, Gao S, Zhao Y,Li P, Liu J, Li P, Tan K and Xie F: Disruption of tumor neovasculature by microbubble enhanced ultrasound: A potential new physical therapy of anti-angiogenesis. Ultrasound Med Biol 38: 253-261, 2012.

11. Hu X, Kheirolomoom A, Mahakian LM, Beegle JR, Kruse DE, Lam KS and Ferrara KW: Insonation of targeted microbubbles produces regions of reduced blood flow within tumor vasculature. Invest Radiol 47: 398-405, 2012.

12. Chen H, Kreider W, Brayman AA, Bailey MR and Matula TJ: Blood vessel deformations on microsecond time scales by ultrasonic cavitation. Phys Rev Lett 106: 034301, 2011.

13. Liu HL, Fan CH, Ting CY and Yeh CK: Combining microbubbles and ultrasound for drug delivery to brain tumors: Current progress and overview. Theranostics 4: 432-444, 2014.

14. Ji L, Si Y, Liu H, Song X, Zhu W and Zhu A: Application of orthogonal experimental design in synthesis of mesoporous bioactive glass. Micropor Mesopor Mat 184: 122-126, 2014.

15. Wang Y, Hu B, Diao X and Zhang J: Antitumor effect of microbubbles enhanced by low frequency ultrasound cavitation on prostate carcinoma xenografts in nude mice. Exp Ther Med 3: 187-191, 2012.

16. Wood AK, Bunte RM, Cohen JD, Tsai JH, Lee WM and Sehgal CM: The antivascular action of physiotherapy ultrasound on a murine tumor: Role of a microbubble contrast agent. Ultrasound Med Biol 33: 1901-1910, 2007.

17. Klibanov A: Microbubble contrast agent: Targeted ultrasound imaging and ultrasound-assisted drug-delivery applications. Invest Radiol 41: 354-362, 2006.

18. Coralic V and Colonius T: Shock-induced collapse of a bubble inside a deformable vessel. Eur J Mech B Fluids 40: 64-74, 2013

19. Gao F, Hu Y and $\mathrm{Hu} \mathrm{H}$ : Asymmetrical oscillation of a bubble confined inside a micro pseudoelastic blood vessel and the corresponding vessel wall stresses. Int J Solids Struct 44: 7197-7212, 2007.

20. Gao F, Xiong C and Xiong Y: Constrained oscillation of a bubble subjected to shock wave in microvessel. Prog Nat Sci 19: $1109-1117,2009$

21. Liu J, Xiao S, Wu S, Ou W, He J, Gao S and Liu Z: Disruption of splenic circulation using microbubble-enhanced ultrasound and prothrombin: A preliminary study. Ultrasound Med Biol 38: 1930-1937, 2012.

22. Moonen $C$ and Lentacker I: Ultrasound assisted drug delivery. Preface. Adv Drug Deliv Rev 72: 1-2, 2014.
23. Sorace AG, Warram JM, Umphrey $H$ and Hoyt $K$ : Microbubble-mediated ultrasonic techniques for improved chemotherapeutic delivery in cancer. J Drug Target 20: 43-54, 2012.

24. Nabili M, Shenoy A, Chawla S, Mahesh S, Liu J, Geist C and Zderic V: Ultrasound-enhanced ocular delivery of dexamethasone sodium phosphate: An in vivo study. J Ther Ultrasound 2: 6, 2014.

25. Kooiman K, Vos HJ, Versluis M and de Jong N: Acoustic behavior of microbubbles and implications for drug delivery. Adv Drug Deliv Rev 72: 28-48, 2014.

26. Wu F, Chen WZ, Bai J, Zou JZ, Wang ZL, Zhu H and Wang ZB: Tumor vessel destruction resulting from high-intensity focused ultrasound in patients with solid malignancies. Ultrasound Med Biol 28: 535-542, 2002.

27. Wood AK, Ansaloni S, Ziemer LS, Lee WM, Feldman MD and Sehgal CM: The antivascular action of physiotherapy ultrasound on murine tumors. Ultrasound Med Biol 31: 1403-1410, 2005.

28. Goertz DE, Todorova M, Mortazavi O, Agache V, Chen B, Karshafian R and Hynynen K: Antitumor effects of combining docetaxel (taxotere) with the antivascular action of ultrasound stimulated microbubbles. PLoS One 7: e52307, 2012.

29. Chen H, Brayman AA, Bailey MR and Matula TJ: Blood vessel rupture by cavitation. Urol Res 38: 321-326, 2010.

30. Ahmadi F, McLoughlin IV, Chauhan S and ter-Haar G: Bio-effects and safety of low-intensity, low-frequency ultrasonic exposure. Prog Biophys Mol Biol 108: 119-138, 2012.

31. Polat BE, Hart D, Langer $R$ and Blankschtein $D$ : Ultrasound-mediated transdermal drug delivery: Mechanisms, scope and emerging trends. J Control Release 152: 330-348, 2011.

32. Carvell KJ and Bigelow TA: Dependence of optimal seed bubble size on pressure amplitude at therapeutic pressure levels. Ultrasonics 51: 115-122, 2011.

33. Stride EP and Coussios CC: Cavitation and contrast: The use of bubbles in ultrasound imaging and therapy. Proc Inst Mech Eng H 224: 171-191, 2010.

34. Hosseinkhah $\mathrm{N}$ and Hynynen $\mathrm{K}$ : A three-dimensional model of an ultrasound contrast agent gas bubble and its mechanical effects on microvessels. Phys Med Biol 57: 785-808, 2012.

35. VanBavel E: Effects of shear stress on endothelial cells: Possible relevance for ultrasound applications. Prog Biophys Mol Biol 93: 374-383, 2007.

36. Dayton P, Klibanov A, Brandenburger G and Ferrara K: Acoustic radiation force in vivo: A mechanism to assist targeting of microbubbles. Ultrasound Med Biol 25: 1195-1201, 1999.

37. Basta G, Venneri L, Lazzerini G, Pasanisi E, Pianelli M, Vesentini N, Del Turco S, Kusmic C and Picano E: In vitro modulation of intracellular oxidative stress of endothelial cells by diagnostic cardiac ultrasound. Cardiovasc Res 58: 156-161, 2003.

38. Chen H, Brayman AA, Kreider W, Bailey MR and Matula TJ: Observations of translation and jetting of ultrasound-activated microbubbles in mesenteric microvessels. Ultrasound Med Biol 37: 2139-2148, 2011.

39. Miller MW, Everbach EC, Cox C, Knapp RR, Brayman AA and Sherman TA: A comparison of the hemolytic potential of Optison and Albunex in whole human blood in vitro: Acoustic pressure, ultrasound frequency, donor and passive cavitation detection considerations. Ultrasound Med Biol 27: 709-721, 2001.

40. Ueda H, Mutoh M, Seki T, Kobayashi D and Morimoto Y: Acoustic cavitation as an enhancing mechanism of low-frequency sonophoresis for transdermal drug delivery. Biol Pharm Bull 32: 916-920, 2009.

41. Tu J, Hwang JH, Matula TJ, Brayman AA and Crum LA: Intravascular inertial cavitation activity detection and quantification in vivo with Optison. Ultrasound Med Biol 32: 1601-1609, 2006. 